\title{
Synthetic Jet Propulsion for Small Underwater Vehicles
}

\author{
AnnMarie Polsenberg Thomas, Michele Milano, Maxwell Grazier G'Sell, Kathleen Fischer, Joel Burdick \\ Division of Engineering and Applied Science \\ California Institute of Technology \\ Pasadena, California 91125 \\ \{ampolsen, milano, maxwell, kathleen, jburdick\}@caltech.edu
}

\begin{abstract}
This paper proposes a new synthetic jet actuation concept for small, low speed, highly maneuverable AUVs. Synthetic jet thrusters, which produce jets of vortex rings, are inspired by the pulsatile jet propulsion of salps, jellyfish, and squid. To assess the potential utility of this scheme, we developed synthetic jet actuator prototypes, and verified their function via both force measurement and flow visualization experiments. We used a genetic-algorithm based technique for optimizing the actuation profile of the thrusters. Also presented is an initial discussion of vehicle design. Our conclusion is that synthetic jet thrusters are a viable propulsion method for small underwater vehicles.
\end{abstract}

Index Terms-autonomous underwater vehicles (AUVs), synthetic jet, vortex ring, optimization, propulsion

\section{INTRODUCTION}

Underwater robots have proven to be an incredibly useful tool for many marine scientists and ecologists. Thus, there is a natural interest in expanding the regimes where they can be used. In particular, there is a growing need for very small, maneuverable, Autonomous Underwater Vehicles (AUVs) that could closely track slow moving animals (such as jellyfish or larvaceans) over extended periods of time (e.g. several days). While such animals are quite simple, their large numbers suggest that they play an important role in marine ecosystems. As discussed in more detail below, it has been difficult to observe such animals with current propeller based AUV and ROV designs for more than a few hours at a time. Thus, their detailed behavior over 24 hours periods is, strictly speaking, still a matter of conjecture. Small, slow, maneuverable AUVs would also be potentially useful for a wide variety of other applications such as detailed small scale surveying, inspection of complex underwater structures, monitoring, and animal tracking.

Underwater robots can be categorized into two main types: autonomous underwater vehicles (AUVs) which operate for relatively long periods without human guidance, and remotely operated vehicles (ROVs) that are powered and teleoperated via a tether connected to a surface command ship. AUVs are our primary interest in this paper. AUVs typically follow the same basic design principle. A propeller thruster is combined with control surfaces (or shrouded thrusters) to propel and steer the vehicle. While this design is incredibly effective for open ocean cruising and large area surveys, it is not very suitable for slow speed tasks such as tracking a slowly moving animal, where subtle maneuvers are required.
ROVs, which are not designed for cruising, typically follow the so-called "Box Design" to achieve better low speed maneuvering and control. These designs sacrifice the low drag body-of-revolution design and add multiple thrusters at different locations and directions to aid maneuverability. Successful ROV designs in this category include WHOI's JASON and MBARI's Tiburon. However, at 7,000 lbs. in weight for Tiburon, these vehicles are not small. Because they are human guided, operator fatigue limits animal tracking time to a few hours. Moreover, high ROV operational costs make longer term observations prohibitively costly. AUV designs that adopt the box design for improved hovering include Stanford's OTTER [1] and WHOI's ABE and SeaBED.

Much work has been done in the area of matching propulsion requirements to the optimal propeller. However, as new regimes (such as slow speed maneuvering of AUVs) are explored, the possibility arises that there may be more desirable propulsion methods for these applications. One potential downside of using propellers can be found during deployment and recovery of most AUVs. As AUVs are typically deployed from a surface ship, the ease with which they are deployed and recovered is often correlated to the condition of the weather and seas. In rough conditions, there is the very real possibility that the vehicle will come into contact with the surface ship. In this situation, any appendages sticking out of the vehicle are at risk of being damaged. A vehicle whose thrusters do not protrude from its body would be more robust to damage during recovery and during maneuvers in tight quarters. Another consideration is that since propellers do not occur in nature, their wake structure may scare animals. We hypothesize that using a propulser with a wake structure more closely resembling that of a sea creature would be less likely to disturb animals (particularly those lacking vision). Moreover, prior experience suggests that slow moving animals can be sucked into the blades of ROVs or AUVs that hover nearby.

In Section II we propose an alternative actuation scheme (a synthetic jet) that addresses many of these concerns. In Section III we summarize the basic mechanics principles of synthetic jet operation and simple models that predict their behavior, while Section IV describes synthetic jet prototypes that we have constructed. To optimize the performance of these devices, we have developed a combined experimental and evolutionary algorithm procedure to empirically find the best operating conditions (Section 
V). We have also subjected these prototypes to qualitative and quantitative flow visualization (Section VI). Finally, Section VII, presents conservative calculations that confirm that synthetic jets are a viable propulsion alternative for small, slow, highly maneuverable AUVs.

\section{OVERVIEW OF APPROACH}

Nature provides many paradigms for underwater propulsion schemes [2], [3]. The inspiration for our proposed propulsion scheme comes from the pulsed jet propulsion method used by many sea creatures: squid, salp and jellyfish are common examples [4], [5], [6]. These creatures propel themselves by pulsing water out of a cavity in their body. As described in more detail below, the fluid rolls up into vortex rings as it exits the body. Weihs shows that a jet consisting of a sequence of vortex rings will exhibit an increased average thrust for a given mass flux relative to what can be accomplished with a steady jet. (It is interesting to note that 70 percent of the roughly 176 known species of cephalopods have mantle lengths of less than $15 \mathrm{~cm}$. This possibly implies pulsatile jet propulsion is best suited to small vehicles, which is the motivating focus of this paper.)

We propose the use of synthetic jets to create pulsative jet streams. Synthetic jets have been used in the aerospace industry for flow control. Currently, the vast majority of researchers studying synthetic jets are looking at applications in air. A main goal of many of these experiments is controlling flow separation on airfoils [7], [8], [9], [10], [11]. In comparison, our goal is to demonstrate that synthetic jets can generate adequate thrust and maneuvering forces for small underwater vehicles.

These jets typically consist of a chamber with a vibrating membrane at one end, and an orifice at the other end [12], [13]. During the membrane's down-stroke, water is drawn into the chamber from around the orifice. During the membrane up-stroke, water is ejected through the orifice, creating a vortex ring (Figure 1). Periodic vibration of the membrane results in a sequence of vortex rings. These rotating rings create a jet-like structure. As discussed in more detail below, this fluid structure results in transferral of net momentum to the fluid, and therefore a propulsive force. We show in Section VII that the thrust required to propel small (25 $\mathrm{cm}$ spherical vehicles) at low speeds (e.g., $10 \mathrm{~cm} / \mathrm{sec}$ ) is very reasonable (less that $0.2 \mathrm{~N}$ ). We demonstrate prototypes below that generate more than this amount of force.

For our purposes, synthetic jets have many potential advantages. First, these jets are compact mechanical structures, requiring only a small volume of space. Hence, when used in small AUVs, they could potentially provide a propulsion scheme which consumes very little of the vehicle's precious internal volume, thereby leaving room for valuable payloads. Second, the synthetic jet has only one moving part, which lowers the probability of mechanical failure. Third, only one simple seal is necessary to seal the actuator from the surrounding fluid. Hence, synthetic jets are ideal for applications where environmental contamination may be an issue. Fourth, synthetic jets can be deployed in such a way that there are no structures protruding into the surrounding flow. Finally, the wake structure and noise generated by synthetic jets are likely to be less obtrusive for underwater surveillance of biological organisms.

Another possible way to use synthetic jets on AUVs is in addition to propellers. As is discussed in many of the references mentioned above, synthetic jets have a history of being used for flow control on airfoils. We hypothesize that synthetic jets could be placed on AUV bodies to modify the vehicle drag and flow separation points. Additionally, the jets could be used for low speed positioning, while propellers are used for quick movement and large area coverage.
A)

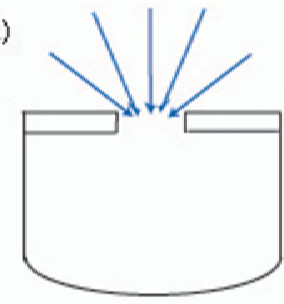

C)

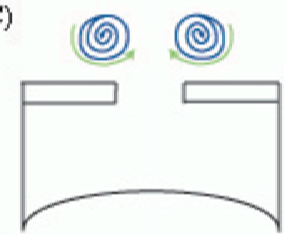

B)

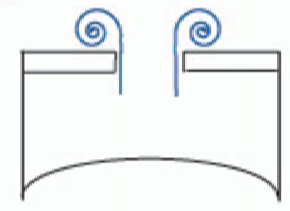

D)

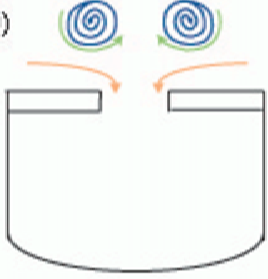

Fig. 1. Stages of a synthetic jet: (A) The initial in-stroke sucks water into the chamber. (B) The out-stroke causes fluid to roll up into a ring. (C) The vortex ring pinches off. (D) During subsequent in-strokes, water is sucked in from around the departing vortex ring.

\section{Physics of Synthetic Jet Flow}

One of the practical goals of this work is to determine the optimal parameters for the design of synthetic jets that optimize thrust or efficiency. Clearly, a greater understanding of the physics of synthetic jet operation and pulsatile jet formation is a prerequisite for design optimization.

In some of the earliest quantitative work, Norbury found that vortex rings can be classified using a single parameter: the non-dimensional mean core radius. This concept set the stage for many methods of characterizing and describing vortex rings [14]. As the goal of this project is to use a synthetic jet to create thrust using vortex ring jets, the process of ring formation is very important. Krueger and Gharib have done studies involving the relationship between vortex ring formation and the thrust of the resulting starting jet. Gharib has found that there seems to be an ideal stroke ratio $(\mathrm{L} / \mathrm{D}=4)$ which allows the maximum circulation to be attained.

It is interesting to note that Weihs points out that L/D of between three and four is a good estimate of what is found 
in salps and cephalopods [6]. (Linden, however, points out some uncertainty in this number, and cites sources putting the L/D of salps anywhere from 6 to 20 . He also highlights the possible problems in trying to fit an L/D model to aquatic creatures, given their flexible chambers [15].) This issue of optimal L/D is important, as it can give a solid starting point for determining the optimal geometry of a synthetic jet. L and D are the length and diameter of the cylindrical slug of fluid ejected from the orifice.

The "slug model" is one promising scheme to model the essential behavior of a synthetic jet. This approach is based on the observation that the "slug" of water that is expelled through the orifice must be converted into vortical motion. This model attempts to predict the size of the vortex ring produced by a given size slug of water, as is shown in Figure 2 [16], [17].
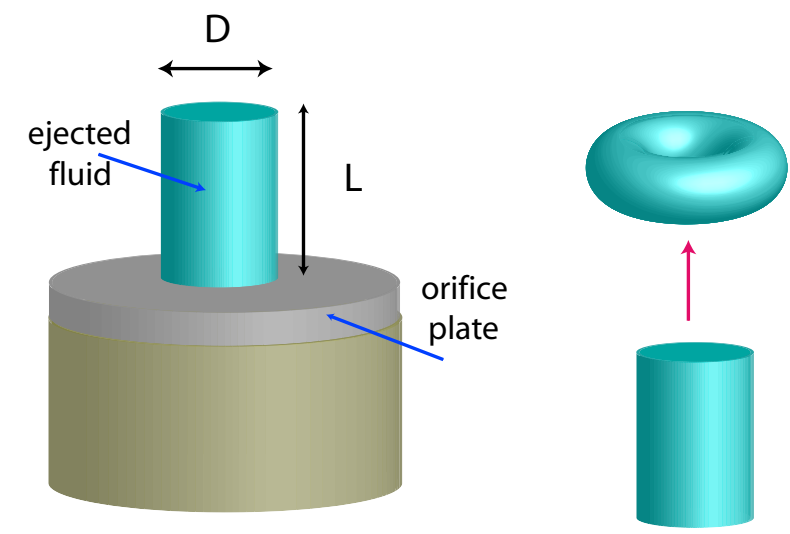

A

B

Fig. 2. The "slug" model: (A) A cylindrical slug of fluid is ejected from the orifice. and (B) the slug rolls up into a vortex ring

One aspect of synthetic jets which is virtually untouched by the above vortex works is the effect that the in-stroke has on the expelled vortex rings. Particular care is needed in characterizing this phenomenon, and experimental observations will play an important role.

As an initial starting point for this project, we attempted to create a preliminary estimate of the force produced by a given synthetic jet. The following uses the "slug model" to approximate the force produced during the out-stroke of the membrane.[18], [19]:

$d_{\text {orifice }}$ : Diameter of jet orifice

$d_{\text {chamber }}$ : Diameter of jet chamber

$d_{d i s k}$ : Diameter of rigid disk attached to the membrane

$\delta$ : Maximum membrane displacement

$U_{m}$ : Velocity of membrane

$U_{p}$ : Intermediate velocity

$U_{e}$ : Velocity at the orifice exit

$R E$ : Reynolds number $\rho$ : Water density

$$
U_{p}=U_{m} * \frac{d_{\text {chamber }}^{2}}{d_{\text {orifice }}^{2}}
$$

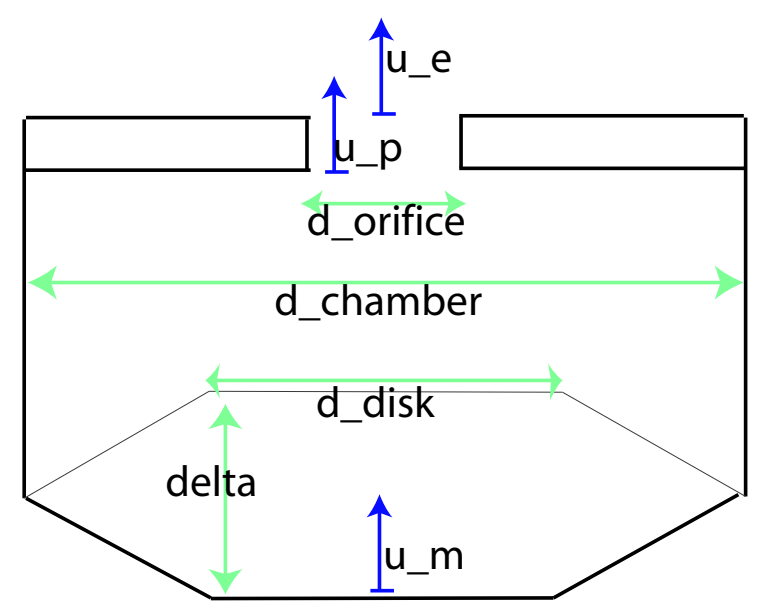

Fig. 3. schematic of synthetic jet

$$
\begin{gathered}
R E=\frac{U_{p} * D_{\text {orifice }}}{\text { viscosity }} \\
U_{m}=2 * \delta * \text { frequency } \\
U_{e}=U_{p} *\left(1+\frac{8}{\sqrt{\pi}} \frac{1}{\sqrt{R E}} \frac{\sqrt{L}}{\sqrt{D}}\right)
\end{gathered}
$$

[18], [20]

Circulation is the line integral of velocity over a line enclosing a vortex; it is a measure of the vortex strength. The reaction force acting on the jet is higher for stronger vortices, so maximizing the circulation is an indirect way of maximizing thrust.

$$
\begin{gathered}
\text { Circulation : } \Gamma=\frac{1}{2} L U_{p} \\
\text { Impulse }: I=\frac{\pi D^{2} \rho}{2} \Gamma
\end{gathered}
$$

If we look only at the outflow of our jet, we can use the impulse to calculate a simple estimate of a lower bound for the average thrust of the jet:

$$
T_{\text {ave }}=\frac{I}{t}
$$

where $t$ is the time of a single outward push.

The reason that this is a fairly crude approximation is that it ignores the interaction between successive rings, as well as the entrainment of fluid by each ring. As we will note later in this paper, we find that a higher frequency of pulsing produces a higher thrust. This implies that the vortex rings produced are closer to each other, thus interacting so as to increase the entrainment effect. For the thrusters that were used in the force measurement experiment described below, $T_{\text {ave }}$ would be on the order of $0.1 \mathrm{~N}$.

It is important to note that the above calculations only account for the out-stroke motion of the membrane. Flow visualizations of synthetic jets show that the vortex rings closest to the orifice are flattened by the in-stroke. We predict that this sucking effect will cause a reduction of thrust. Unfortunately, it has proven difficult to predict just 
how great this effect will be. This difficulty in modeling highlights the importance of experimental study of synthetic jets.

\section{Synthetic Jet Prototype}

In order to study both the flow patterns created by the jets, as well as to measure the forces produced by them, it was necessary to design and build synthetic jet prototypes. We designed a system that is as modular as possible so that we can easily change parameters and quantify their effects. There are four main parts of our system, which can be seen in Figure 4:

- orifice plate

- fluid chamber

- flexible membrane

- actuator

Fluid in the chamber is ejected through a cylindrical hole in the center of the orifice plate to create the vortex ring jet. The geometry of the orifice plate sets the length and diameter of the ejected fluid slug. Currently, we have designed our orifice plates to realize an L/D ratio of 4 for the reasons described above. It is likely that a cylindrical hole may not be the best shape for optimizing thrust, and we hope to later perform experiments on the effects of an orifice with angled sides.

Our prototypes use a cylindrical chamber geometry. Historical studies of nozzle flow suggests that the jets may be more efficient if the chamber tapers toward the orifice. We hope to perform experiments on this geometry in the future.

The membrane is a thin (1/32 inch) rubber sheet that is attached to the actuator via a metal disk that is glued to the membrane's underside.

We have experimented with a few different actuators. The results presented in this paper are based on voice coil actuators: a Bruel and Kjael 4810 minishaker (designed to vibrate small objects), and a voice coil from a consumergrade audio speaker. Voice coil actuators allowed a large degree of control over the membrane's driving waveforms. Unfortunately, the voice coils do not have a large throw, nor is their geometry or size optimized for our application. Consequently, the prototypes shown below were optimized for research flexibility, and not for compactness. In the longer term, as the important design parameters become well understood, we anticipate that other actuation schemes can drive the diaphragm in a more compact physical package. (roughly $1 / 4$ or $1 / 5$ of the sizes shown here). Our preliminary work suggests that solenoids can provide greater diaphragm displacement and greater force in a smaller package.

\section{Force Measurement And Optimizations}

While the fluid models reviewed above provide a qualitative and quantitative understanding of the key parameters influencing synthetic jet performance, the detailed performance of an actuator will depend upon many additional variables whose influence can be difficult to quantify

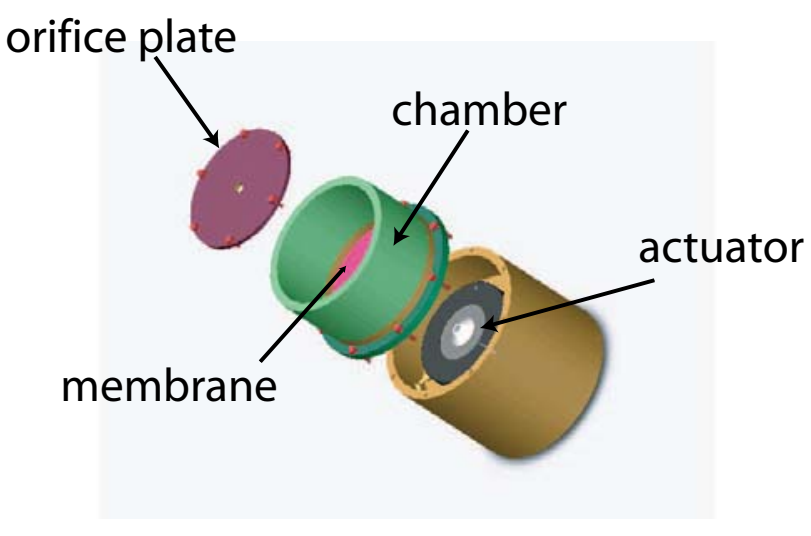

Fig. 4. Solidworks image of thruster

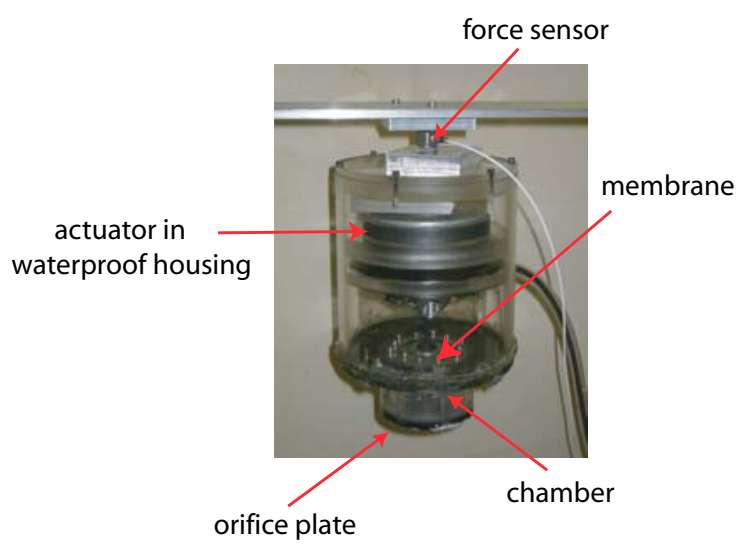

Fig. 5. Synthetic Jet prototype

exactly. For example, roughness of the surfaces in contact with the fluid, the geometry of the fluid chamber, the flexibility of the membrane, etc. will all make subtle changes in an actuator's performance. Hence, guided by the modeling scheme above, we developed a more empirical approach to find a "good" or "optimal" set of actuator parameters. Our method combines an evolutionary algorithm with an automated experimental apparatus to find the best free parameters within a fixed mechanical implementation. For a fixed mechanical implementation (i.e., fixed orifice hole, chamber, diaphragm, and voice coil geometry), the frequency, amplitude and velocity profile of the membrane's movement can still be varied. It is our observation that changing these parameters can have a significant effect on the thrust produced by a synthetic jet. In order to maximize this thrust, we used a genetic algorithm to optimize over the space of possible choices of these parameters. These combinations were used to command the synthetic jet actuators. The resulting average thrust force was measured, and these actual experimental measurements were then used the by genetic algorithm that is described below.

To measure the force produced by the various parameter combinations, we attached the thruster to a force sensor (Kistler 9212 force sensing load cell) that was mounted on 
a rigid test-stand that supported the thruster in a tank of water (as shown in Figure 5). We then measured the force produced by the given parameters.

Review of genetic algorithm. The genetic algorithm used in this paper is a modification of the Controlled Random Search (CRS) method of [21]. For convenience, a brief summary is provided (see [22] for details). Let $G(\lambda)$ be a cost function to be minimized by the choice of design parameters, $\lambda \in R^{n}$. For this work, $\frac{1}{G}$ is the average thrust produced by the synthetic jet (produced over a 20 second period). In a first phase, a set of $S$ parameters vectors, or "population points," are initially chosen at random according to a uniform distribution within a defined $n$-dimensional search. The inequality $S \gg n$ must hold for the algorithm to work properly. Then the algorithm proceeds as follows:

1) Find the parameter $\lambda_{\max }$ from the initial set of $S$ points which maximizes $G: \lambda_{\max }=$ $\arg \left[\max _{i=1, \ldots, M} G\left(\lambda_{i}\right)\right] ; G_{\max }=G\left(\lambda_{\max }\right)$;

2) Randomly choose $n+1$ different population points: $\lambda_{1}, \ldots, \lambda_{n+1}$ (breeding set). All subsequent operations are performed on this set;

3) Mutation step: for all breeding set points, with probability

$P_{i}=\left(1-\alpha^{I}\right) \cdot\left(1-\beta^{\frac{\left(G\left(\lambda_{i}\right)-G_{T}\right)}{\bar{G}}}\right) \cdot \gamma$, replace the point $\lambda_{i}$ with one randomly chosen within the search volume limits;

4) Recombination step: for each of the $n+1$ points determine the centroid, $\lambda_{i}$, of the other $n$ points, i.e. $\underline{\lambda_{i}}=\frac{1}{n} \sum_{j=1}^{n} \lambda_{j}$

4.a) Generate offspring $\lambda_{s i}=2 \underline{\lambda_{i}}-\lambda_{n+1}$; if $\lambda_{s i}$ is not contained in the search volume, process next point in breeding set ;

4.b) Calculate $G\left(\lambda_{s i}\right)$ : if $G\left(\lambda_{s i}\right)<G_{\max }$ then purge $\lambda_{\max }$ from population, and substitute by offspring $\lambda_{s i}$;

5) Compute the new $G_{\max }$, if necessary;

6) Iterate steps 4 and 5 on the whole breeding set;

7) If convergence test fails, return to step 1.

There are 4 parameters $\left(\alpha, \beta, \gamma, G_{T}\right.$ and 2) and two variables $(I$ and $\bar{G})$ that must be chosen by the user in this optimization scheme. The variable $I$ is the number of consecutive iterations in which the population has not changed. It provides an empirical measure of the need for fresh information through increased mutation probability. The variable $\bar{G}$ is the average population fitness, used as a scaling factor. The most important parameter is $G_{T}$, a threshold value used for the convergence test: we declare convergence once all of the population's fitness values are below it. With this convergence criterion, population points will be clustered inside the domain defined as: $\left\{\lambda \mid G(\lambda)<G_{T}\right\}$. According to the formula defining mutation probability, the parameter $0<=\alpha<=1$ modulates the mutation rate during the optimization process, and the parameter $0<=\gamma<=1$ enforces its upper bound, since $0<=\beta<=1$. The term containing the parameter $\beta$ causes population members far from convergence (with fitness

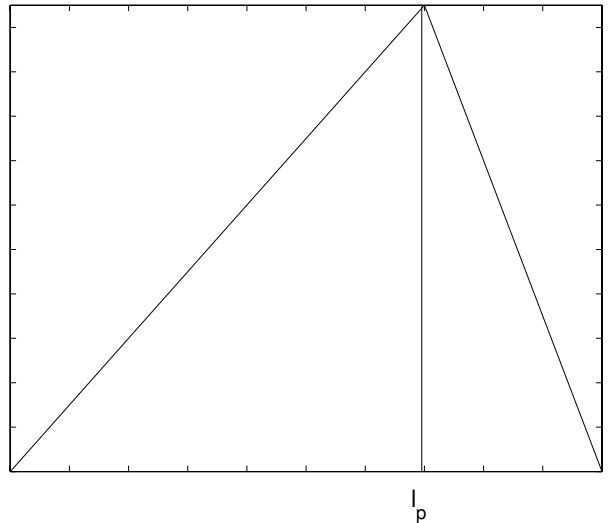

Fig. 6. Parametrization for the outlet velocity, showing outlet velocity amplitude (ordinates) as a function of time (abscissa), for a single period of duration $T$. The vertical line marks the point $l_{p}$, where the velocity attains its maximum amplitude $A$.

$>G_{T}$ ) to mutate more frequently. (CRS can be regarded as a GA with zero mutation probability.)

In order to minimize the effect of measurements and environmental noise on the accuracy of the fitness function value, we repeated each experiment 5 times, averaging the corresponding fitness values.

We parametrized the outlet velocity (which is directly a function of the driving diaphragm's movement) as a periodic sawtooth function (see Fig. 6), whose shape was described by three parameters: the peak amplitude $A$, the frequency $f$, and the location of the peak inside the period, $l_{p}$. The parameter $l_{p}$ ranged from 0 to 1 , with smaller values denoting a profile peak closer to the beginning of the period. This parametrization defines a 3-dimensional search space in which it would be impractical to perform an exhaustive search (since each experiment lasted slightly more than one minute). Hence, the evolutionary algorithm described above is a much more suitable procedure to find good parameter choices.

We used a GA population of 50 elements, with the parameters $\alpha$ and $\beta$ fixed to 0.25 , the upper bound $\gamma$ to 0.02 and the threshold $G_{T}$ to 0.1 , which is small enough to ensure a sufficient exploration of the search volume. This algorithm yielded a satisfactory result in about 1000 iterations.

The final population produced by the GA showed that the maximum force that can be produced by this synthetic jet (the voice coil "stinger" version) is approximately 0.25 $\mathrm{N}$. It should be noted that we will estimate, in the vehicle discussion section, that the force required to move the vehicle at projected maximum speed is only $0.118 \mathrm{~N}$. The force of 0.25 Newtons was produced using maximum power. Thus, we need less power to travel at the speeds we project our vehicle to regularly operate at.

Interestingly, we found that the optimal waveform to send to the thruster was one that caused the membrane to pull in quickly and push out slowly. We found that thrust increases proportionally with frequency. In our setup the highest thrust was achieved at a frequency of $20 \mathrm{Hertz}$, but 
as this was the maximum frequency we tested it is possible that the optimal frequency is even higher.

\section{Flow Visualization}

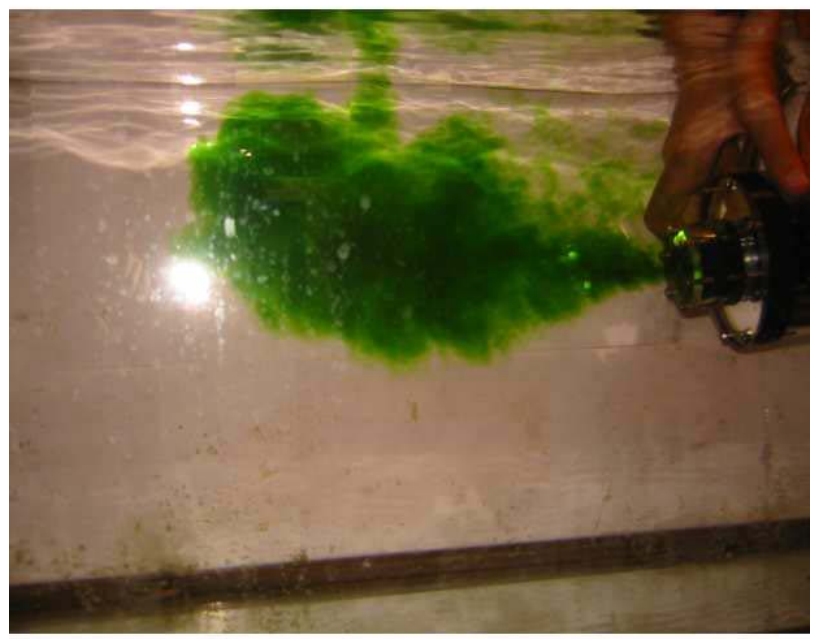

Fig. 7. Dye flow visualization of synthetic jet thruster operation.

One important element in our experimental work is the visualization of the fluid flow. As very little work has been done studying synthetic jets in water, there is much to be learned about the shapes of the flow structures created. We deployed both quantitative and qualitative flow visualization at a range of operating parameters. The qualitative data was obtained expelling colored dye from the actuator chamber, and using a high speed camera to capture the gross flow phenomena. It was very clear from these qualitative efforts that well defined vortex rings are created, and that the overall flow structure is a jet (see Figure 7).

More quantitative flow data was collected using Digital Particle Image Velocimetry (DPIV). DPIV proceeds by seeding water with small reflective particles. A laser sheet is used to illuminate the flow, and a video camera repeatedly photographs the sheet. (For this experiment we used a $150 \mathrm{~mW}$ New Wave Gemini laser and photographed 30 frames a second.) From the video stream, cross-correlation of the particle locations in consecutive frames can be used to determine the local flow velocity. These values are then used to estimate an overall velocity field. Subsequently, the vorticity field can be calculated from the velocity field [23].

Our DPIV experiments were conducted on the voice coil actuated synthetic jet. We imaged the flow with the laser sheet oriented along the jet axis (the horizontal plane), and with the sheet oriented perpendicular to this axis. The water was seeded using 14 micron particles. Figure 8 shows a vorticity plot from a horizontal plane visualization of our synthetic jet actuated at 6 Volts and $10 \mathrm{~Hz}$. Pairs of counterrotating vortices are clearly visible in the field. In the near and intermediate field of the orifice, the flow has a clear vortex ring jet structure. In the far field, notice that the axial flow breaks up (the "blooming phenomena" [24]).

\section{Vorticity of DPIV measurements for one of the optimal cases}

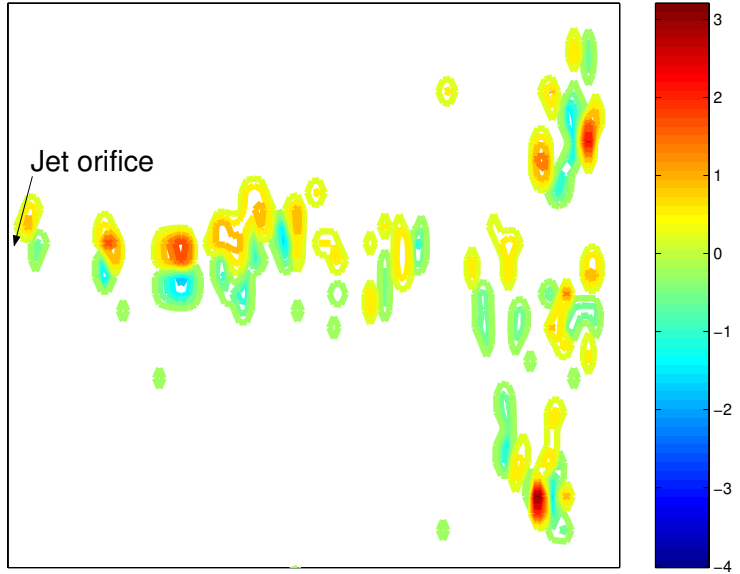

Fig. 8. DPIV vorticity plot of synthetic jet flow. Color coded according to vorticity strength (positive vorticity means a clockwise rotating vortex and vice versa)

\section{Thrust REQUiREMENTS FOR CANDIDATE VEHICLE DESIGN}

As discussed above, one of our primary goals is to build a vehicle which is capable of tracking slow moving sea creatures (e.g. jellyfish) that swim at speeds between 2 and $10 \mathrm{~cm} / \mathrm{sec}$. Since these creatures drift with the ambient current, our vehicle need not be able to swim against the current. To aid stability in the vertical plane, while maximizing mobility in the horizontal plane, we propose a oblate spheroidal vehicle shape. This allows for four horizontal thrusters and two vertical thrusters.
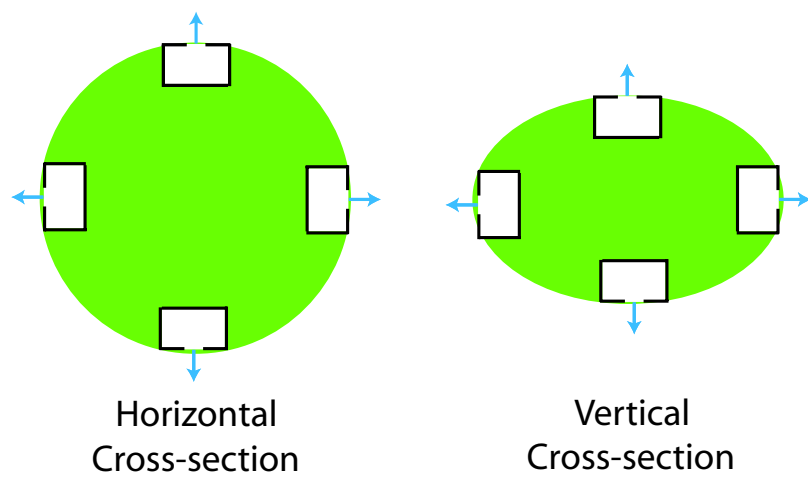

Fig. 9. Cross sections of the proposed vehicle and thruster configuration.

Our preliminary investigation seeks to demonstrate that synthetic jets can provide viable propulsion and maneuvering forces for such a vehicle. To roughly estimate the amount of force needed to move this vehicle, we can conservatively approximate its shape as a sphere. (The drag for the "flying saucer" shape will be less than the drag of a sphere. Thus the following calculations are a worst case scenario.)

$$
\operatorname{Drag}=C_{D} \frac{1}{2} U^{2} \rho S
$$


where $U$ is the vehicle speed, $\rho$ the density of water, and $C_{D}$ is the drag coefficient. The drag coefficient in term can be found as a function of the ambient flow's Reynolds number, $R e=U D / \nu$, where $D$ is the vehicle diameter (m) and $\nu$ is the kinematic viscosity of water.

For speeds between 0.02 and $0.1 \frac{\mathrm{m}}{\mathrm{s}}$ the Reynolds number of a $0.25 \mathrm{~m}$ diameter sphere ranges from 4,717 to 23,585 . In this range $C_{D}$ is approximately 0.47 . Hence, movement of the vehicle at the speeds requires an upper bound of between 0.0047 and $0.118 \mathrm{~N}$ of force (see Figure 10). Note that for the thruster configurations shown in Figure 9, additional forces can be generated by simultaneous actuator of two thrusters. Various combinations of thrusters as well as pulsatile frequency allow a large range of motion on the vehicle's part. While we don't foresee our initial vehicle needing to go faster than the maximum speed possible with one thruster actuating alone, it is promising to know that higher speeds are achievable through the use of multiple thrusters.

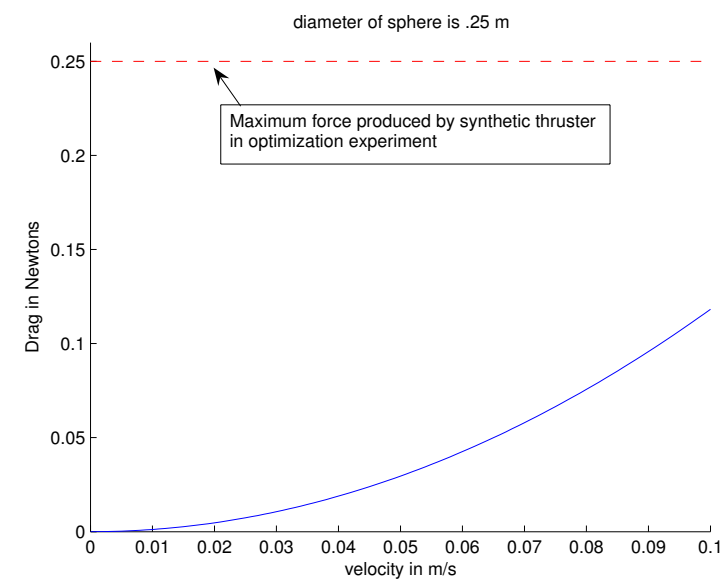

Fig. 10. Vehicle drag as a function of velocity

\section{FUTURE WORK}

The next steps in our project is to construct the vehicle proposed above and begin exploring the control issues it will present. We hypothesize that by varying the frequency, amplitude, and thrusting sequences of the jets we will be capable of highly precise maneuvering. Another component that we are actively pursuing is the further characterization of the flows produced by submerged synthetic jets. (For example, some of our DPIV data hints at the possibility of "blooming" occurring. Learning to control this slightly deleterious effect will allows us to harness even more of the thrust produced by these jets.)

Based on the above results and observations, we are confident that synthetic jets are a reasonable propulsion system for the category of small, low speed, highly maneuverable AUVs that we are interested in developing.

\section{ACKNOWLEDGMENT}

This work could not have been accomplished without the assistance of Professor Mory Gharib of Caltech. We would like to thank Vicente Fernandez, Aimee Eddins, Jesse Escobeda, and Joanna Cohen for their work on designing and building the thrusters. John Dabiri provided help with the vortex modeling. The first author was supported by a Department of Defense fellowship. Partial support was also provided by the National Science Foundation (NSF9402726).

\section{REFERENCES}

[1] H. Wang, R. Marks, T. McLain, S. Fleischer, D. Miles, G. Sapilewski, and S. Rock, "Otter: A testbed submersible for robotics research," in ANS 1995, Monterey, CA, 1995.

[2] M. Denny, Air and Water: The Biology and Physics of Life's Media. Princeton University Press, 1993.

[3] S. Vogel, Cats' Paws and Catapults: Mechanical Worlds of Nature and People. New York: W.W. Norton and Company, 1998.

[4] J. Seikmann, "On a pulsating jet from the end of a tube, with applications to the propulsion of certain aquatic animals," Journal of Fluid Mechanics, vol. 15, pp. 399-418, 1963.

[5] E. Trueman, "Motor performance of some cephalopods," Journal of Experimental Biology, vol. 49, pp. 495-505, 1968.

[6] D. Weihs, "Periodic jet propulsion of aquatic creatures," Fortschritte der Zoologie, vol. 24, pp. 171-175, 1977.

[7] F.-J. Chen, C. Yao, R. Bryant, and R. Fox, "Development of synthetic jet actuators for active flow control at nasa langley," AIAA Paper 2000-2405, 2000.

[8] A. Crook, A. Sadiri, and N. Wood, "The development and implementation of synthetic jets for the control of seperated flow," in 17th Applied Aerodynamics Conference, Norfolk, VA, 1999.

[9] Q. Gallas, R. Holman, T. Nishada, B. Carroll, M. Sheplak, and L. Cattafesta, "Lumped element modeling of piezoelectric-driven synthetic jet actuators," AIAA Journal, vol. 41, no. 2, pp. 240-247, 2003.

[10] R. James, J. Jacobs, and A. Glezer, "A round turbulent jet produced by an oscillating diaphragm," Physics of Fluids, vol. 8, no. 9, pp. 2484-2495, 1996.

[11] S. Mallinson, G. Hong, and J. Reizes, "Some characteristics of synthetic jets," in 30th AIAA Fluid Dynamics Conference, Norfolk, VA, 1999.

[12] A. Glezer and M. Amitay, "Synthetic jets," Annual Review of Fluid Mechanics, vol. 34, pp. 503-529, 2002.

[13] B. Smith and A. Glezer, "Vectoring and small-scale motions effected in free shear flows using synthetic jet actuators," AIAA Paper 970213, 1997.

[14] J. Norbury, "A family of steady vortex rings," Journal of Fluid Mechanics, vol. 57, pp. 417-431, 1973.

[15] P. Linden and J. Turner, "The formation of 'optimal' vortex rings, and the efficiency of propulsion devices," Journal of Fluid Mechanics, vol. 427, pp. 61-72, 2001.

[16] K. Mohseni and M. Gharib, "A model for universal time scale of vortex ring formation," Physics of Fluids, vol. 10, no. 10, pp. 24362438, 1998.

[17] K. Mohseni, R. Hongyu, and T. Colonius, "Numerical experiments on vortex ring formation," Journal of Fluid Mechanics, vol. 430, pp. 267-282, 2001.

[18] M. Shusser, M. Rosenfeld, and K. Mohseni, "On the effect of pipie boundary layer growth on the formation of a laminar vortex ring generated by a piston/cylinder arrangement," Theoretical and Computational Fluid Dynamics, vol. 15, pp. 306-316, 2002.

[19] K. Shariff and A. Leonard, "Vortex rings," Annual Review of Fluid Mechanics, vol. 24, pp. 235-279, 1992.

[20] J. Dabiri and M. Gharib, "A revised slug model boundary layer correction for starting jet vorticity flux," Theoretical and Computational Fluid Dynamics, vol. 17, pp. 293-295, 2004.

[21] W. Price, "A controlled random search procedure for global optimization," The Computer Journal, vol. 20, no. 4, 1976.

[22] M. Milano, Machine Learning Techniques for Flow Modeling and Control. Swiss Federal Institute of Technology, 2002.

[23] C. Willert and M. Gharib, "Digital particle image velocimetry," Experiments in Fluids, vol. 10, pp. 181-193, 1991.

[24] W. Reynolds, D. Parekh, P. Juvet, and M. Lee, "Bifurcating and blooming jets," Annual Review of Fluid Mechnics, vol. 35, pp. 295315, 2003. 